\title{
Decreased Trigeminal Sensitivity in Anosmia
}

\author{
Hilmar Gudziola Michael Schubert ${ }^{a}$ Thomas Hummel ${ }^{b}$ \\ a Department of Otorhinolaryngology, Friedrich Schiller University of Jena Medical School, Jena, and \\ bDepartment of Otorhinolaryngology, University of Dresden Medical School, Dresden, Germany
}

\section{Key Words}

Nociception, chemosensory · Olfaction · Irritation • Multisensory integration

\begin{abstract}
The present study aimed to investigate intranasal trigeminal sensitivity in a large sample of patients with anosmia due to different etiologies. We investigated the trigeminal detection threshold for formic acid in healthy controls $(n=96)$ and patients with anosmia due to head trauma $(n=18)$ or sinonasal disease $(n=54)$. Anosmics exhibited higher thresholds compared with normosmics $(p<0.001)$. In addition, thresholds were found to be higher in patients with posttraumatic anosmia compared to anosmics with sinonasal disease $(p<0.001)$. The data indicate that (1) loss of olfactory sensitivity in humans may be associated with a decreased sensitivity towards trigeminal stimuli and (2) alteration of intranasal trigeminal function is stronger in patients with posttraumatic anosmia compared to patients with sinonasal disease. This may have implications for the medicolegal investigation of anosmic patients where trigeminal stimuli are frequently used to assess the patient's response bias.

\section{Introduction}

Most odorants produce not only olfactory but also trigeminally mediated sensations $[1,2]$. Numerous reports relate to interactions between the two major intranasal chemosensory systems, the olfactory and the trigeminal nerves. For example, it has been shown that at certain concentrations trigeminal activation may suppress olfactorily mediated sensations [3]. On the other hand, research also indicates that olfactory activation increases sensitivity to trigeminally mediated stimuli $[4,5]$. This has been shown on an electrophysiological level where patients with anosmia exhibit smaller responses to trigeminal stimuli as indicated by EEG-derived event-related potentials [6]. On a behavioral level, respiratory reflexes to intranasal trigeminal stimuli are found to be significantly faster and stronger in normosmics compared to anosmics [7]. And finally, clinical observations [8,9] indicate that olfactory loss is frequently accompanied by a decreased responsiveness to trigeminal stimulation.

This work was conducted at the Department of Otorhinolaryngology, Friedrich Schiller University of Jena Medical School, Jena, Germany.

\begin{tabular}{ll}
\hline KARGER & ( 2001 S. Karger AG, Basel \\
Fax +41 61 306 1234 & 0301-1569/01/0632-0072\$17.50/0 \\
$\begin{array}{l}\text { E-Mail karger@karger.ch } \\
\text { www.karger.com }\end{array}$ & $\begin{array}{l}\text { Accessible online at: } \\
\text { www.karger.com/journals/orl }\end{array}$
\end{tabular}

Thomas Hummel, MD

Department of Otorhinolaryngology, University of Dresden Medical School Fetscherstrasse 74

D-01307 Dresden (Germany)

Tel. +49 351458 4189, Fax +49 351458 4326, E-Mail thummel@rcs.urz.tu-dresden.de 
However, these changes of trigeminal sensitivity in relation to olfactory activation are still a matter of debate [10]. Thus, the aim of the present study was to address this question at the threshold level. As most of the previous work was performed in relatively small groups the present study was designed to use larger samples. This approach would also allow to check for possible differences between patients with anosmia due to different etiologies, i.e. patients with posttraumatic anosmia and anosmic patients with sinonasal diseases.

\section{Materials and Methods}

The study was performed according to the Declaration of Helsinki (Summerset West amendment) on guidelines for biomedical research involving human subjects. All subjects/patients provided written consent after they were thoroughly acquainted with all details of the investigation. All testing was performed in a well-ventilated room; room temperature was approximately $21^{\circ} \mathrm{C}$.

\section{Formic Acid Used as a Trigeminal Stimulant}

Formic acid was selected as stimulant for its strong trigeminal impact. Preliminary experiments have already revealed that a concentration of $1 \% \mathrm{v} / \mathrm{v}$ formic acid (analytical grade; Merck, Darmstadt, Germany; solvent: deionized water) was above the trigeminal threshold; stimuli at this concentration were clearly perceived as irritating.

\section{Trigeminal Impact of Formic Acid}

Before commencement of tests in anosmic patients, the trigeminal impact of this concentration of formic acid was assessed in 14 healthy controls (10 men, 4 women; age range $18-64$ years). To this end, an experimental design was applied as previously described [11, 12] where trigeminal activation is quantified by the subjects'/ patients' ability to lateralize stimuli presented to either the left or right nostril. The formic acid stimulus was presented to either one nostril in a high-density polyethylene squeeze bottle (total volume $250 \mathrm{ml}$ ) filled with $30 \mathrm{ml}$ of $1 \% \mathrm{v} / \mathrm{v}$ formic acid; at the same time an identical bottle filled with $30 \mathrm{ml}$ solvent (deionized water) was presented to the contralateral nostril. The bottles had a pop-up spout that was placed close to the nostril. A puff of approximately $30 \mathrm{ml}$ air was delivered to each nostril by pressing the two bottles at the same time. A total of 32 formic acid stimuli were delivered to the blindfolded subjects/patients at an interstimulus interval of approximately $30 \mathrm{~s}$; stimulation of the left or right nostril followed a pseudorandomized sequence. Following each stimulus subjects/patients were asked to identify the nostril where formic acid had been presented. In this experiment all subjects/patients were able to identify the site of stimulation; the median of correct identifications was 25.5 (range: 21-31 correct identifications out of 32 trials; chance level: 16 correct identifications). This clearly indicated that the currently used concentration of formic acid is a potent stimulant of the intranasal trigeminal system.

\section{Participants}

In 72 anosmics (47 men, 25 women; age range 19-73 years; mean age 43 years, SD 14 years) trigeminal thresholds to formic acid were
Table 1. Quantification of olfactory sensitivity

\begin{tabular}{lc}
\hline & Points \\
\hline Butanol detection threshold, \% v/v & 5 \\
0.002 & 4 \\
0.005 & 3 \\
0.016 & 2 \\
0.049 & 1 \\
$0.15-0.44$ & 0 \\
$1.3-4.0$ & 5 \\
\hline Identification rate, \% & 4 \\
$90-100$ & 3 \\
$70-80$ & 2 \\
$50-60$ & 1 \\
$30-40$ & 0 \\
$10-20$ & \\
0 & \\
\hline & \\
The subjects'/patients' olfactory sensitiv- \\
ity was evaluated in relation to scores re- \\
ceived for their performance in both the \\
odor detection threshold task and the odor \\
identification test (maximum score 10, mini- \\
mum score 0). All anosmics scored <2, all \\
normosmics scored >8. \\
\hline
\end{tabular}

compared to those of 96 normosmics ( 60 men, 36, women; age range 20-88 years; mean age 45 years, SD 15 years). Normosmics and anosmics did not differ significantly in terms of age ( $\mathrm{t}$ test: $\mathrm{t}=0.83$, d.f. $=166, p=0.41)$ or gender (Mann-Whitney test: $Z=0.37, p=$ $0.71)$. In 54 patients ( 22 men, 32 women) anosmia was caused by sinonasal disease; in 18 patients ( 15 men, 3 women) anosmia was due to head trauma.

\section{Assessment of Olfactory Sensitivity}

All of the participating subjects/patients underwent olfactory testing similar to procedures described by Cain et al. [13]. First, using the method of ascending limits n-butanol detection thresholds were measured in the blind-folded subjects/patients (double-forced-choice paradigm); threshold criterion was the correct identification of $n$ butanol 4 times in a row. Then, odor identification was tested for 10 common odors (camphor, lemon, skatole, perfume, amyl acetate, coffee, cinnamon, cloves, vanillin and gas) using a list with 20 descriptors. All odorants were released immediately in front of the subjects'/patients' nostrils from brown glass bottles (total volume $50 \mathrm{ml}$ ). Olfactory sensitivity was quantified on a scale from 0 to 10 points (table 1). All anosmics scored $\leq 1$ point, normosmics scored $\geq 9$ points.

\section{Irritation Thresholds}

Lateralized irritation thresholds for formic acid were assessed similar to the n-butanol thresholds described above. Formic acid was presented in ascending concentrations which had been arranged in a quasi-logarithmic manner (table 2). Subjects/patients were in- 


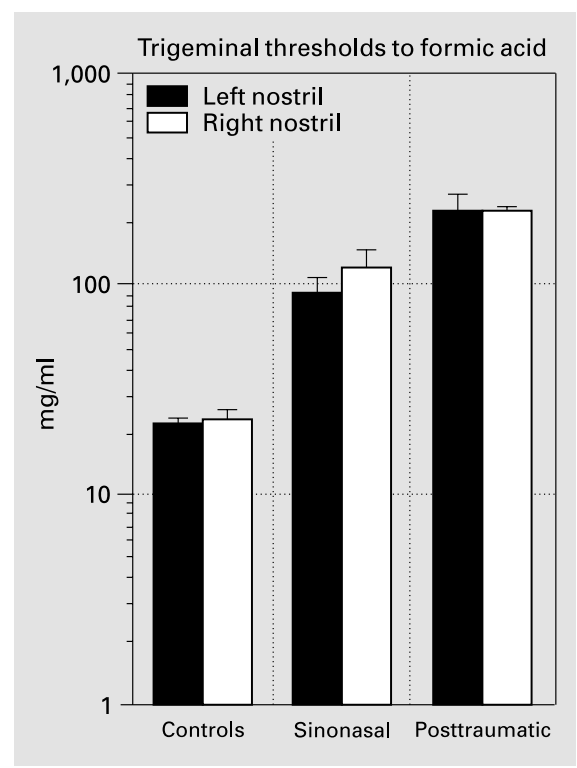

Fig. 1. Trigeminal thresholds for formic acid (means, SEM) in patients with posttraumatic anosmia $(n=18)$, anosmia caused by sinonasal disease $(n=54)$ and controls $(n=96)$ separately for the left (closed bars) and right nostril (open bars). Thresholds differed significantly $(\mathrm{p}<0.001)$ between the 3 groups.

Table 2. Concentrations of formic acid used to assess trigeminal detection thresholds

\begin{tabular}{lc}
\hline $\begin{array}{l}\text { Dilution } \\
\text { number }\end{array}$ & $\begin{array}{l}\text { Concentration } \\
\mathrm{mg} / \mathrm{ml}\end{array}$ \\
\hline 1 & 1 \\
2 & 2 \\
3 & 4 \\
4 & 8 \\
5 & 12 \\
6 & 41 \\
7 & 82 \\
8 & 204 \\
9 & 407 \\
10 & 815 \\
11 & 1,220 \\
\hline
\end{tabular}

structed to indicate the presence of trigeminally mediated sensations like burning or stinging; had this been indicated 4 times in a row the respective concentration was assumed to be the trigeminal threshold. It was noted down as the concentration of formic acid in milligrams per milliliter. Subjects/patients closed either one nostril with the tip of the thumb. Opened bottles were presented approximately $2 \mathrm{~cm}$ in front of the other nostril. Testing of the left or right nostril was randomized across subjects/patients.

\section{Statistical Analyses}

Results were analyzed by means of SPSS 9.0 for Windows ${ }^{\mathrm{TM}}$. Data were submitted to analyses of variance (repeated measures design) with 'nostril' as within-subject factor and 'group' as betweensubject factor; degrees of freedom were adjusted according to Greenhouse-Geisser. Post hoc testing was performed using Bonferroni tests. The alpha level was set at 0.05 .

\section{Results}

Comparison of trigeminal thresholds indicated a significant effect of the factor 'group' $[F(2,165)=35.2$, $p<$ $0.001]$ with anosmics having higher thresholds. No difference between nostrils could be observed $[F(1,1,665)=$ $0.62, p=0.43]$. Bonferroni post hoc tests revealed significant threshold differences $(\mathrm{p}<0.001)$ between normosmics, anosmics with posttraumatic disorder and anosmics with sinonasal disorder (fig. 1).

\section{Discussion}

The present results indicate that anosmics do have higher trigeminal thresholds compared to normosmic controls. Importantly, this seems to be independent of the cause of the anosmia as both anosmics with sinonasal disease and anosmics with posttraumatic olfactory loss exhibit similar differences compared to normosmic subjects.

The differences between normosmics and anosmics are in line with previous reports indicating decreased trigeminal sensitivity in anosmia. Specifically, this has been observed clinically [8], using both psychophysical [14] and behavioral measures of trigeminal function [7], and by means of electrophysiological techniques [6]. The present results also compare to those of Walker and Jennings [15], who reported an increase in nasal irritation thresholds for acetic acid, propionic acid and amyl acetate in anosmics. In addition, similar findings had already been obtained in experimental animals [16-18]. In this context, however, it has to be noted that the presently used approach to assess responsiveness in the trigeminal system certainly has weaknesses as it is confounded by odorous sensations elicited by formic acid. Nevertheless, assuming that both subjects and patients strictly adhered to instructions and reported only the presence of trigeminally mediated sensations, the present data clearly indicate the presence of differences between controls and anosmics who had approximately 10 -fold higher thresholds. Thus, the present data can be viewed as part of a 
series of experiments indicating lower trigeminal sensitivity in anosmia.

What could be the reason for such decreased trigeminal sensitivity? One reason might be sought in an interaction between the olfactory and the intranasal trigeminal system. Specifically, on a suprathreshold level, Kobal and co-workers $[4,5]$ demonstrated in normosmics that trigeminal stimuli are perceived as more intense when they were accompanied by olfactory stimulation; both $\mathrm{H}_{2} \mathrm{~S}$ and vanillin produced an increase in the perceived intensity of $\mathrm{CO}_{2}$ stimuli. These interactions may take place in the thalamus (e.g. rat: mediodorsal nucleus [19]), higherorder CNS sites [20-22] or at the mucosa. Analogous to the concept that olfactory responses might be modified by an axon reflex of trigeminal afferents lying in the olfactory epithelium [23-25], it should also be considered that olfactory afferent activity may influence trigeminal input via neurosecretory changes.

Other reasons may relate to the cause of the olfactory loss. In posttraumatic anosmia one could imagine CNS lesions which may affect the processing of trigeminally mediated sensations. What argues against this is that the present results indicated decreased trigeminal function in both posttraumatic olfactory loss and loss of olfactory function following sinonasal disease. However, while olfactory loss may be generally accompanied by a certain decrease in trigeminal sensitivity, the present data may also indicate an additional effect in patients with head trauma as they exhibited significantly higher trigeminal thresholds compared to patients with sinonasal disorder.

Decreased trigeminal sensitivity in anosmia also has consequences for medicolegal investigations in anosmic patients. Here, many otorhinolaryngologists use trigeminal stimuli as probes to investigate the patient's bias to report intranasally perceived sensations. In case the patient does not respond to trigeminal stimuli such as mustard oil or acetic acid, clinically this is often interpreted as an indicator of malingering. Considering that anosmics may have higher trigeminal thresholds, this practice needs to be reconsidered.

\section{References}

1 von Skramlik E: Handbuch der Physiologie der niederen Sinne. Leipzig, Thieme, 1926.

2 Doty RL, Brugger WPE, Jurs PC, Orndorff MA, Snyder PJ, Lowry LD: Intranasal trigeminal stimulation from odorous volatiles: Psychometric responses from anosmic and normal humans. Physiol Behav 1978;20:175-185.

3 Cain WS, Murphy C: Interaction between chemoreceptive modalities of odour and irritation. Nature 1980;284:255-257.

4 Kobal G, Hummel C: Cerebral chemosensory evoked potentials elicited by chemical stimulation of the human olfactory and respiratory nasal mucosa. Electroencephalogr Clin Neurophysiol 1988;71:241-250.

5 Livermore A, Hummel T, Kobal G: Chemosensory event-related potentials in the investigation of interactions between the olfactory and the somatosensory (trigeminal) systems. Electroencephalogr Clin Neurophysiol 1992;83: 201-210.

6 Hummel T, Barz S, Lötsch J, Roscher S, Kettenmann B, Kobal G: Loss of olfactory function leads to a decrease of trigeminal sensitivity. Chem Senses 1996;21:75-79.

7 Kendall-Reed M, Walker JC: Human respiratory responses to irritants. Talk presented at the 'Workshop on Sensory Irritation', Sea Lodge, La Jolla, May 1998.

8 Kobal G, Hummel T: Effects of flupirtine on the pain-related evoked potential and the spontaneous EEG. Agents Actions 1988;23:117119.
9 Van Toller S: Assessing the impact of anosmia: Review of a questionnaire's findings. Chem Senses 1999;24:705-712.

10 Cometto-Muniz JE, Cain WS, Abraham MH, Kumarsingh R: Sensory properties of selected terpenes: Thresholds for odor, nasal pungency, nasal localization, and eye irritation. Ann NY Acad Sci 1998;855:648-651.

11 Kobal G, Van Toller S, Hummel T: Is there directional smelling? Experientia 1989;45: 130-132.

12 Berg J, Hummel T, Huang G, Doty RL: Trigeminal impact of odorants assessed with lateralized stimulation. Chem Senses 1998;23:587.

13 Cain WS, Gent JF, Goodspeed RB, Leonard G: Evaluation of olfactory dysfunction in the Connecticut Chemosensory Clinical Research Center (CCCRC). Laryngoscope 1988;98:83-88.

14 Wysocki CJ, Cowart BJ, Varga E: Nasal-trigeminal sensitivity in normal aging and clinical populations. Chem Senses 1997;22:826.

15 Walker JC, Jennings RA: Comparison of odor perception in humans and animals; in Laing DG, Doty RL, Breipohl W (eds): The Human Sense of Smell. Berlin, Springer, 1991, pp 261280.

16 Silver WL, Mason JR, Marshall DA, Maruniak JA: Rat trigeminal, olfactory, and taste responses after capsaicin desensitization. Brain Res 1986;333:45-54.

17 Henton WW, Smith JC, Tucker D: Odor discrimination in pigeons following section of the olfactory nerves. J Comp Physiol Psychol 1969; 69:317-323.
18 Walker JC, Tucker D, Smith JC: Odor sensitivity mediated by the trigeminal nerve in the pigeon. Chem Senses Flav 1979;4:107-116.

19 Inokuchi A, Kimmelmann CP, Snow JB Jr: Convergence of olfactory and nasotrigeminal inputs on possible trigeminal contributions to olfactory responses in rat thalamus. Eur Arch Otorhinolaryngol 1993;249:473-477.

20 Stone $\mathrm{H}$ : Effect of ethmoidal nerve stimulation on olfactory bulbar electrical activity; in Pfaffmann C (ed): Olfaction and Taste. New York, Rockefeller University Press, 1969, pp 216220.

21 Stone H, Rebert CS: Observations on trigeminal olfactory interactions. Brain Res 1970;21: 138-142.

22 Hummel T, Yousem DM, Alsop DC, Geckle RJ, Doty RL: Functional MRI of olfactory and intranasal chemosensory trigeminal nerve activation. Soc Neurosci Abstr 1997;23:2076.

23 Bouvet JF, Delaleu JC, Holley A: Olfactory receptor cell function is affected by trigeminal nerve activity. Neurosci Lett 1987;77:181186.

24 Bouvet JF, Delaleu JC, Holley A: The activity of olfactory receptor cells is affected by acetylcholine and substance P. Neurosci Res 1988;5: 214-223.

25 Finger TE, Getchell ML, Getchell TV, Kinnamon JC: Affector and effector functions of peptidergic innervation of the nasal cavity; in Green BG, Mason JR, Kare MR (eds): Chemical Senses: Irritation. New York, Dekker, 1990, pp 1-20. 